\title{
DIGITALCOMMONS
}

\section{Comparisons of Two Quantile Regression Smoothers}

Rand Wilcox

University of Southern California, rwilcox@usc.edu

Follow this and additional works at: http://digitalcommons.wayne.edu/jmasm

\section{Recommended Citation}

Wilcox, Rand (2016) "Comparisons of Two Quantile Regression Smoothers," Journal of Modern Applied Statistical Methods: Vol. 15 : Iss. 1 , Article 5.

DOI: $10.22237 /$ jmasm/1462075440

Available at: http://digitalcommons.wayne.edu/jmasm/vol15/iss1/5 


\section{Invited Article Comparisons of Two Quantile Regression Smoothers}

\section{Rand R. Wilcox}

University of Southern California

Los Angeles, CA

The small-sample properties of two non-parametric quantile regression estimators are compared. The first is based on constrained B-spline smoothing (COBS) and the other is based on a variation and slight extension of a running interval smoother. $\mathrm{R}$ functions for applying the methods were used in conjunction with default settings for the various optional arguments. Results indicate that the modified running interval smoother has practical value. Manipulation of the optional arguments might impact the relative merits of the two methods, but the extent to which this is the case remains unknown.

Keywords: Running interval smoother, COBS, Harrell-Davis estimator, LOWESS, Well Elderly 2 study, depressive symptoms, perceived control

\section{Introduction}

Consider the problem of estimating and plotting a regression line when the goal is to determine the conditional quantile of some random variable $Y$ given $X$. Quantile regression methods have been studied extensively and plots of the regression line can provide a useful perspective regarding the association between two variables. One approach is to assume that the conditional $q^{\text {th }}$ quantile of $Y$, given $X$, is given by

$$
Y_{q}=\beta_{0}+\beta_{1} X
$$

where $\beta_{0}$ and $\beta_{1}$ are unknown parameters. For the special case where the goal is to estimate the median of $Y$, given $X$, least absolute regression can be used, which predates least squares regression by about a half century. A generalization, aimed

Dr. Wilcox is Professor of Psychology at the University of Southern California. Email himat:rwilcox@usc.edu. 
at dealing with any quantile, was derived by Koenker and Bassett (1978). While the assumption of a straight regression line appears to provide a good approximation of the true regression line in various situations, this is not always the case. One strategy for dealing with any possible curvature is to use some obvious parametric model. For example, add a quadratic term. But generally this can be unsatisfactory, which has led to the development of nonparametric regression lines, often called smoothers (e.g., Härdle, 1990; Efromovich, 1999; Eubank, 1999; Györfi, Kohler, Krzyzk, \& Walk, 2002). For the particular case where the goal is to model the conditional quantile of $Y$, given $X$, one way of dealing with curvature in a reasonably flexible manner is to use constrained B-spline smoothing (COBS). The many computational details are summarized in Koenker and $\mathrm{Ng}$ (2005); see in particular section 4 of their paper. The Koenker-Ng method improves on a computational method studied by $\mathrm{He}$ and $\mathrm{Ng}$ (1999), and builds upon results in Koenker, $\mathrm{Ng}$, and Portnoy (1994). Briefly, let $\rho_{\mathrm{q}}(u)=\mathrm{u}(q-\mathrm{I}(u<0))$, where the indicator function $\mathrm{I}(u<0)=1$ if $u<0$; otherwise $\mathrm{I}(u<0)=0$. The goal is to estimate the $q^{\text {th }}$ quantile of $Y$ given $X$ by finding a function $\omega(X)$ that minimizes

$$
\sum_{i} \rho_{q}\left(Y_{i}-\omega\left(X_{i}\right)\right)
$$

based on the random sample $\left(X_{1}, Y_{1}\right), \ldots,\left(X_{n}, Y_{n}\right)$. The estimate is based on quadratic B-splines with the number of knots chosen via a Schwartz-type information criterion. Here, COBS is applied via the R package cobs.

The motivation for this study stems from the use of COBS when analyzing data from the Well Elderly 2 study (Jackson et al., 2009; Clark et al., 2012). A general goal was to assess the efficacy of an intervention strategy aimed at improving the physical and emotional health of older adults. A portion of the study dealt with understanding the association between cortisol and various measures of stress and wellbeing. Before and six months following the intervention, participants were asked to provide, within 1 week, four saliva samples over the course of a single day, to be obtained on rising, $30 \mathrm{~min}$ after rising but before taking anything by mouth, before lunch, and before dinner. Extant studies (e.g., Clow et al., 2004; Chida \& Steptoe, 2009) indicated measures of stress are associated with the cortisol awakening response, which is defined as the change in cortisol concentration that occurs during the first hour after waking from sleep. CAR is taken to be the cortisol level upon awakening minus the level of cortisol after the participants were awake for about an hour. 


\section{COMPARISONS OF TWO QUANTILE REGRESSION SMOOTHERS}

After intervention (with a sample size of 328), COBS indicated some seemingly unusually shaped regression lines. One of these had to do with the association between CAR and a measure of depressive symptoms using the Center for Epidemiologic Studies Depressive Scale (CESD). The CESD (Radloff, 1977) is sensitive to change in depressive status over time and has been successfully used to assess ethnically diverse older people (Lewinsohn, Hoberman, \& Rosenbaum, 1988; Foley, Reed, Mutran, \& DeVellis, 2002). Higher scores indicate a higher level of depressive symptoms. Figure 1 shows the estimated regression line for males when $q=0.5$. (There were 157 males.) The estimated regression line for $q=0.75$ had a shape very similar to the one shown in Figure 1.

Another portion of the study dealt with the association between CAR and a measure of perceived control. Perceived control was measured with the instrument in Eizenman, Nesselroade, Featherman, and Rowe (1997). The scores ranged between 16 and 32 and consisted of a sum of Likert scales. Now the 0.75 quantile regression line appears as shown in Figure 2. Again, there was concern about the shape of the regression line.

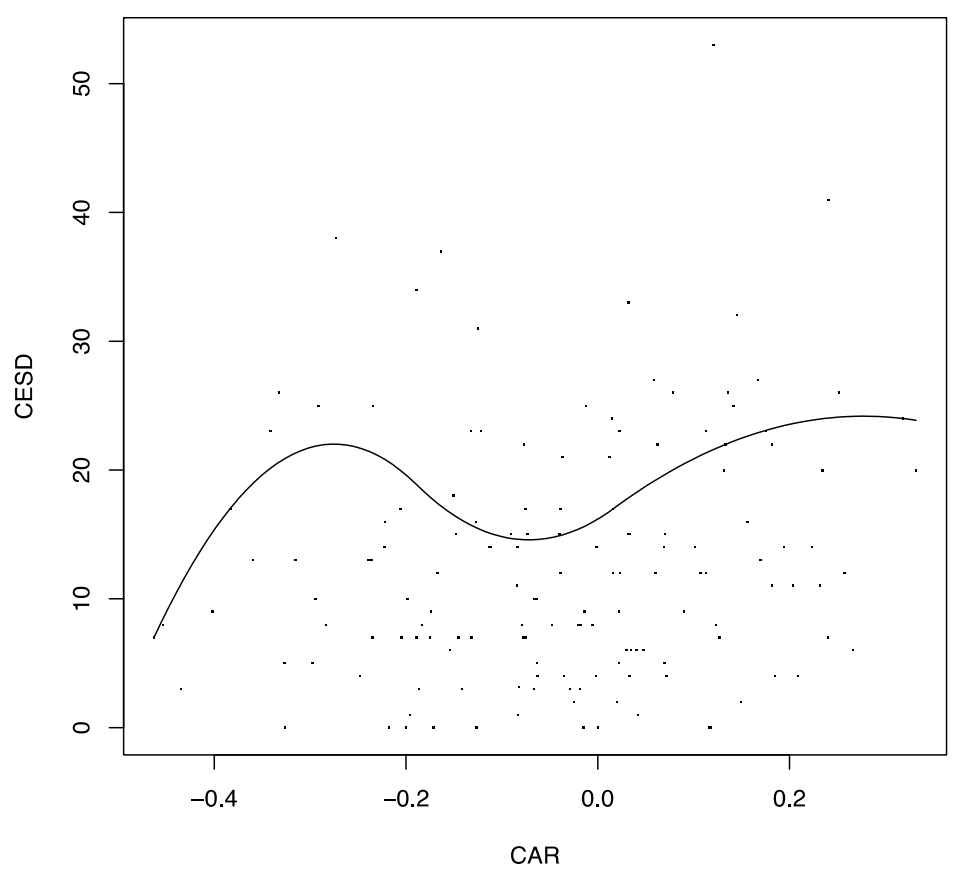

Figure 1. COBS regression line for predicting the 0.5 quantile of CESD, for males, based on the cortisol awakening response after intervention 
RAND R. WILCOX

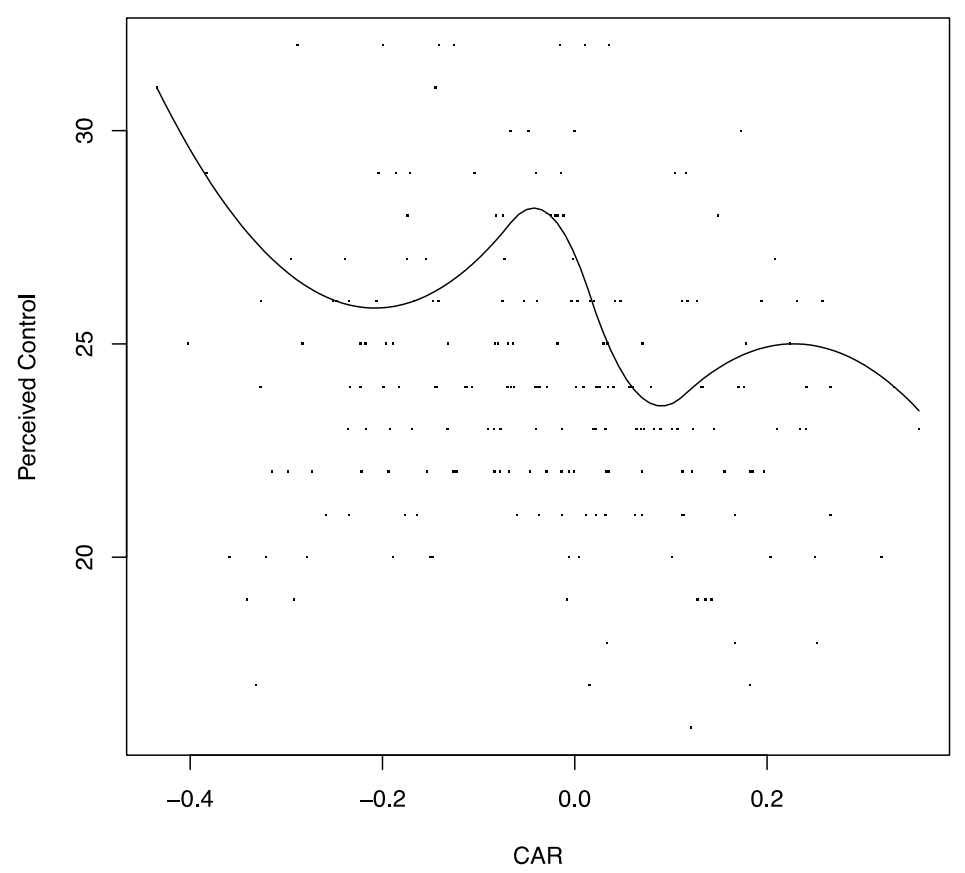

Figure 2. COBS regression line for predicting the 0.75 quantile of perceived control based on the cortisol awakening response

One possibility is that the regression lines in Figures 1 and 2 are a reasonable approximation of the true regression. But another possibility is that they reflect a type of curvature that poorly approximates the true regression line. Suppose that

$$
Y=\beta_{0}+\beta_{1} X+\lambda(X) \varepsilon
$$

where $\lambda(X)$ is some function used to model heteroscedasticity and $\varepsilon$ is a random variable having mean zero and variance $\sigma^{2}$. Some preliminary simulation results suggested that if $\beta_{0}=0, \beta_{1}=1$, and both $X$ and $\varepsilon$ have standard normal distributions, reasonably straight regression lines are obtained using COBS. However, if $\varepsilon$ has a skewed light-tailed distribution (a $g$-and- $h$ distribution, details of which are described in a later section) and if, for example, $\lambda(X)=|X|+1$, instances are encountered where a relatively high degree of curvature is encountered. An example is given in Figure 3 with $n=100$. 


\section{COMPARISONS OF TWO QUANTILE REGRESSION SMOOTHERS}

These results motivated consideration of an alternative quantile regression estimator. A few checks suggested that the problems just illustrated are reduced considerably, but there are no systematic simulation results providing some sense of how this alternative estimator compares to COBS. Consequently, the goal in this paper is to compare these estimators in terms of bias and mean squared error. Two additional criteria are used. The first is the maximum absolute error between the predicted and actual quantile being estimated. The other is aimed at characterizing how the estimators compare in terms of indicating a monotonic association when in fact one exists. This is done via Kendall's tau between the predicted and true quantiles.

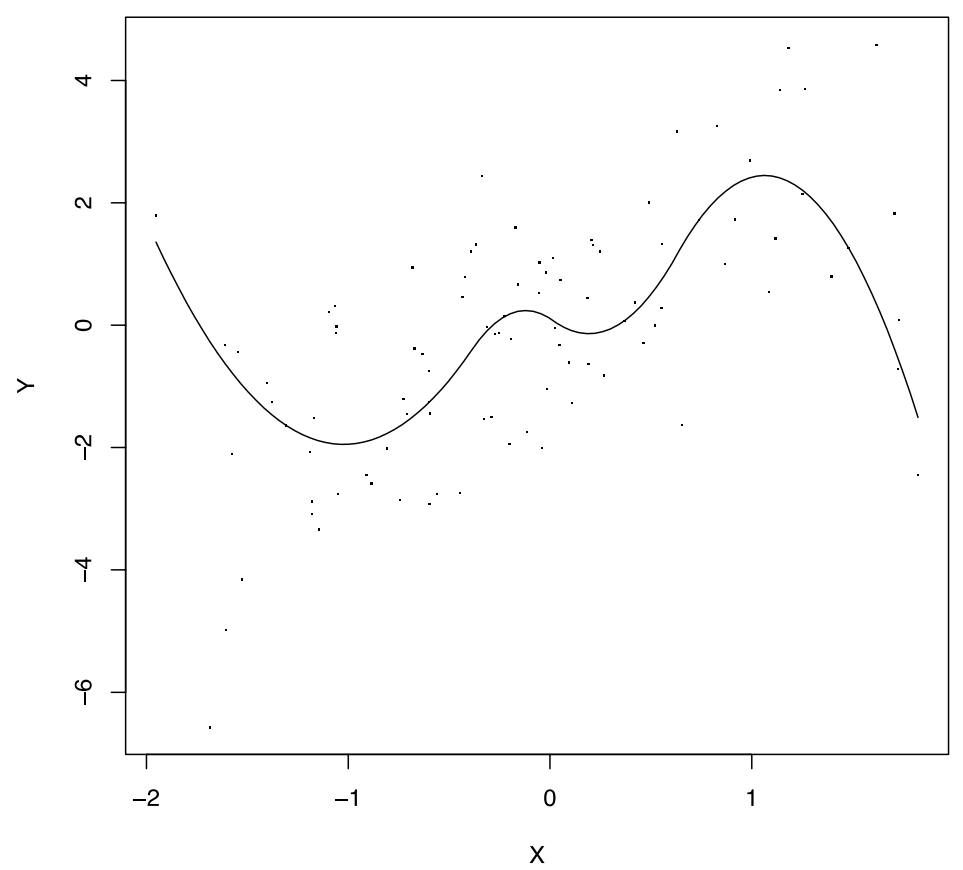

Figure 3. COBS regression line for predicting the 0.5 quantile using generated data, $n=100$

It is noted that COBS is being applied using the $\mathrm{R}$ package cobs in conjunction with default settings for the various arguments. The argument lambda alters how the regression line is estimated and might possibly improve the fit to data via visual inspection. But obviously this strategy is difficult to study via simulations. The 


\section{RAND R. WILCOX}

alternative estimator used here is applied with an R function (qhdsm), again using default settings for all of the arguments. The performance of the method is impacted by the choice for the span (the constant $f$ in later sections). The simulations reported here provide information about the relative merits of the two estimators with the understanding that perhaps their relative merits might be altered based on a judgmental process that goes beyond the scope of this paper.

The following section provides the details of the alternative estimator. Later sections report simulation results comparing COBS to the alternative estimator and illustrate the difference between the two estimators for the data used in Figures 13.

\section{Alternative Estimator}

The alternative estimator consists of a blend of two smoothers: the running interval smoother (e.g., Wilcox, 2012) and the smoother derived by Cleveland (1979), typically known as LOWESS. The running interval has appeal because it is readily adapted to any robust estimator. In particular, it is easily applied when the goal is to estimate the conditional quantile of $Y$ given $X$. However, often this smoother gives a somewhat jagged looking plot of the regression line. Primarily for aesthetic reasons, this issue is addressed by further smoothing the regression line via LOWESS.

The version of the running interval smoother used here is based in part on the quantile estimator derived by Harrell and Davis (1982). The Harrell-Davis estimate of the $q^{\text {th }}$ quantile uses a weighted average of all the order statistics. Let $Z_{1}, \ldots, Z_{n}$ be a random sample, let $U$ be a random variable having a beta distribution with parameters $a=(n+1) q$ and $b=(n+1)(1-q)$, and let

$$
w_{i}=\mathrm{P}\left(\frac{i-1}{n} \leq U \frac{i}{n}\right) \text {. }
$$

The estimate of the $q^{\text {th }}$ quantile is

$$
\hat{\theta}_{q}=\sum w_{i} Z_{(i)}
$$

where $Z_{(1)} \leq \ldots \leq Z_{(n)}$ are the $Z_{1}, \ldots, Z_{n}$ written in ascending order. Here the focus is on estimating the median and the 0.75 quantile. That is, $q=0.5$ and 0.75 are used. 


\section{COMPARISONS OF TWO QUANTILE REGRESSION SMOOTHERS}

In terms of its standard error, Sfakianakis and Verginis (2006) show that the Harrell-Davis estimator competes well with alternative estimators that again use a weighted average of all the order statistics, but there are exceptions. Sfakianakis and Verginis derived alternative estimators that have advantages over the HarrellDavis in some situations, but when sampling from heavy-tailed distributions, the standard error of their estimators can be substantially larger than the standard error of $\hat{\theta}_{q}$. Comparisons with other quantile estimators are reported by Parrish (1990), Sheather and Marron (1990), as well as Dielman, Lowry, and Pfaffenberger (1994). The only certainty is that no single estimator dominates in terms of efficiency. For example, the Harrell-Davis estimator has a smaller standard error than the usual sample median when sampling from a normal distribution or a distribution that has relatively light tails, but for sufficiently heavy-tailed distributions, the reverse is true (Wilcox, 2012, p. 87).

The running interval smoother is applied as follows: Let $\left(X_{1}, Y_{1}\right), \ldots,\left(X_{n}, Y_{n}\right)$ be a random sample from some unknown bivariate distribution, and let $f$ be some constant to be determined. Then the point $x$ is said to be close to $X_{i}$ if

$$
\left|X_{i}-x\right| \leq f \times \mathrm{MADN}
$$

where MADN is MAD/0.6745, MAD is the median of $\left|X_{1}-M\right|, \ldots,\left|X_{n}-M\right|$, and $M$ is the usual sample median based on $X_{1}, \ldots, X_{n}$. For normal distributions, MADN estimates the standard deviation, in which case $x$ is close to $X_{i}$ if $x$ is within $f$ standard deviations of $X_{i}$. Let

$$
\mathrm{N}\left(X_{i}\right)=\left\{j:\left|X_{j}-X_{i}\right| \leq f \times \mathrm{MADN}\right\}
$$

That is, $\mathrm{N}(X)$ indexes the set of all $X_{j}$ values that are close to $X_{i}$. Let $\hat{\theta}_{i}$ be the Harrell-Davis estimate based on the $Y_{j}$ values such that $j \in \mathrm{N}\left(X_{i}\right)$. To get a graphical representation of the regression line, compute $\hat{\theta}_{i}$, the estimated value of $Y$ given that $X=X_{i}, i=1, \ldots, n$, and then plot the points $\left(X_{i}, \hat{\theta}_{1}\right), \ldots,\left(X, \hat{\theta}_{n}\right)$. Typically $f=0.8$ or 1 gives good results, but of course exceptions are encountered. Here, $f=0.8$ is assumed unless stated otherwise.

As previously indicated, the plot produced by the running interval smoother can be a bit ragged. Consequently, the initial smooth was smoothed again by proceeding as follows: Given $X_{j}$, let $\delta_{i}=\left|X_{i}-X_{j}\right|, i=1, \ldots, n$. 


\section{RAND R. WILCOX}

Sort the $\delta_{i}$ values and retain the $\xi_{n}$ pairs of points that have the smallest $\delta_{i}$ values, where $\xi$ is a number between 0 and 1 and plays the role of a span. Here, $\xi=0.75$ is used. Let $\delta_{m}$ be the largest $\delta_{i}$ value among the retained points. Let

$$
Q_{i}=\frac{\left|X_{j}-X_{i}\right|}{\delta_{m}}
$$

and, if $0 \leq Q_{i}<1$, set

$$
w_{i}=\left(1-Q_{i}^{3}\right)^{3}
$$

Otherwise, set $w_{i}=0$. Next, use weighted least squares to predict $\hat{\theta}_{j}$ corresponding to $X$ using the $w_{i}$ values as weights. That is, determine the values $b_{1}$ and $b_{0}$ that minimize

$$
\sum w_{i}\left(\hat{\theta}_{i}-b_{0}-b_{i} X_{i}\right)^{2}
$$

and estimate $\hat{\theta}_{j}$ with $\tilde{\theta}_{j}=b_{0}+b_{1} X_{j}$. The final plot of the quantile regression is taken to be the line connecting the points $\left(X_{j}, \tilde{\theta}_{j}\right)^{2}(j=1, \ldots, n)$. This will be called method $\mathrm{R}$ henceforth.

\section{Simulation}

Simulations were used to compare the small-sample properties of COBS and the modified running interval smoother based on $K=4000$ replications and sample size $n=50$. The data were generated according to the model

$$
Y=X+\lambda(X) \varepsilon
$$

where $X$ is taken to have a standard normal distribution and $\varepsilon$ has one of four distributions: normal, symmetric and heavy-tailed, asymmetric and light-tailed, and asymmetric and heavy-tailed. More precisely, the distribution for the error term was taken to be one of four $g$-and- $h$ distributions (Hoaglin, 1985) that contain the 


\section{COMPARISONS OF TWO QUANTILE REGRESSION SMOOTHERS}

standard normal distribution as a special case. Let $Z$ be a random variable that has a standard normal distribution, and let

$$
W=\frac{\exp (g Z)-1}{g} \exp \left(h Z^{2} / 2\right)
$$

unless $g=0$, in which case

$$
W=Z \exp \left(h \frac{Z^{2}}{2}\right)
$$

Then $W$ has a $g$-and- $h$ distribution, where $g$ and $h$ are parameters that determine the first four moments. The four distributions used here were the standard normal $(g=h=0.0)$, a symmetric heavy-tailed distribution $(h=0.2, g=0.0)$, an asymmetric distribution with relatively light tails $(h=0.0, g=0.2)$, and an asymmetric distribution with heavy tails $(g=h=0.2)$. Table 1 shows the skewness $\left(\kappa_{1}\right)$ and kurtosis $\left(\kappa_{2}\right)$ for each distribution. Hoaglin (1985) summarizes additional properties of the $g$-and- $h$ distributions.

Table 1. Some properties of the $g$-and- $h$ distribution

\begin{tabular}{rrrr}
$\mathbf{g}$ & $\mathbf{h}$ & $\mathbf{k 1}$ & $\mathbf{k 2}$ \\
\hline 0.00 & 0.00 & 0.00 & 3.00 \\
0.00 & 0.20 & 0.00 & 21.46 \\
0.20 & 0.00 & 0.61 & 3.68 \\
0.20 & 0.20 & 2.81 & 155.98 \\
\hline
\end{tabular}

Three choices for $\lambda$ were considered: $\lambda \equiv 1, \lambda=|X|+1$, and $\lambda=1 /(|X|+1)$. These three choices are henceforth called VP 1, 2, and 3, respectively.

Note that based on how the data are generated, as indicated by (5), ideally a smoother should indicate a monotonic increasing association between $X_{1}, \ldots, X_{n}$ and $\tilde{\theta}_{1}, \ldots, \tilde{\theta}_{n}$, where $\tilde{\theta}_{i}$ is the estimate of the $q^{\text {th }}$ quantile of $Y$, given that $X=X_{i}$, based on either COBS or method R. The degree to which this goal was accomplished was measured with Kendall's tau.

Details about the four criteria used to compare COBS and method $\mathrm{R}$ are as follows: The first criterion was mean squared error, which was estimated with 


\section{RAND R. WILCOX}

$$
\frac{1}{n K} \sum_{k=1}^{K} \sum_{i=1}^{n}\left(\theta_{i k}-\tilde{\theta}_{i k}\right)^{2}
$$

where now, for the $k^{\text {th }}$ replication, $\theta_{i k}$ is the true conditional $q^{\text {th }}$ quantile of $Y$ given $X=X_{i}$. Bias was estimated with

$$
\frac{1}{n K} \sum_{k=1}^{K} \sum_{i=1}^{n} \theta_{i k}-\tilde{\theta}_{i k}
$$

The third criterion was the mean maximum absolute error:

$$
\frac{1}{K} \sum_{k=1}^{K} \max \left\{\left|\theta_{1 k}-\tilde{\theta}_{1 k}\right|, \ldots,\left|\theta_{n k}-\tilde{\theta}_{n k}\right|\right\}
$$

The fourth criterion was

$$
\frac{1}{K} \sum \tau_{k}
$$

where, for the $k^{\text {th }}$ replication, $\tau_{k}$ is Kendall's tau between $X_{1}, \ldots, X_{n}$ and $\tilde{\theta}_{1}, \ldots, \tilde{\theta}_{n}$.

It is noted that the $\theta_{i k}$ values are readily determined because the transformation used to generate observations from a $g$-and- $h$ distribution is monotonic and quantiles are location and scale equivariant.

Simulation results are reported in Tables 2 and 3, where RMSE is the mean squared error of COBS divided by the mean squared error of method R, and RMAX is the maximum absolute value of COBS divided by the maximum absolute value of the error based on method R. As can be seen, generally method R competes well with COBS in terms of RMSE and RMAX, but neither method dominates. For $q=0.5, \mathrm{R}$ is uniformly better in terms of RMSE, but for $q=0.75$ and VP 3 , COBS performs better than R. As for RMAX, R performs best for VP 1 and 2, while for VP 3 the reverse is true. Bias for both methods is typically low, with COBS seeming to have an advantage over method $\mathrm{R}$. The main result is that in terms of $\tau$, method $\mathrm{R}$ dominates. That is, the simulations indicate that method $\mathrm{R}$ is better at avoiding an indication of curvature that does not reflect the true regression line, as was the case in Figure 3. 


\section{COMPARISONS OF TWO QUANTILE REGRESSION SMOOTHERS}

Table 2. Simulation results for $q=0.5$

\begin{tabular}{|c|c|c|c|c|c|c|c|c|}
\hline \multirow[b]{2}{*}{$g$} & \multirow[b]{2}{*}{$h$} & \multirow[b]{2}{*}{ VP } & \multirow[b]{2}{*}{ RMSE } & \multirow[b]{2}{*}{ RMAX } & \multicolumn{2}{|c|}{ BIAS } & \multicolumn{2}{|c|}{$\boldsymbol{T}$} \\
\hline & & & & & COBS & $\mathbf{R}$ & COBS & $\mathbf{R}$ \\
\hline 0.0 & 0.0 & 1 & 1.284 & 1.293 & 0.002 & 0.002 & 0.957 & 0.997 \\
\hline 0.0 & 0.0 & 2 & 1.405 & 2.050 & -0.002 & -0.002 & 0.773 & 0.927 \\
\hline 0.0 & 0.0 & 3 & 1.281 & 0.726 & -0.001 & -0.001 & 0.989 & 1.000 \\
\hline 0.0 & 0.2 & 1 & 1.160 & 1.333 & -0.002 & -0.002 & 0.955 & 0.994 \\
\hline 0.0 & 0.2 & 2 & 1.395 & 2.076 & 0.005 & 0.009 & 0.794 & 0.917 \\
\hline 0.0 & 0.2 & 3 & 1.104 & 0.753 & -0.003 & -0.002 & 0.991 & 1.000 \\
\hline 0.2 & 0.0 & 1 & 1.247 & 1.292 & 0.015 & 0.031 & 0.954 & 0.996 \\
\hline 0.2 & 0.0 & 2 & 1.400 & 2.048 & 0.023 & 0.035 & 0.786 & 0.930 \\
\hline 0.2 & 0.0 & 3 & 1.220 & 0.732 & 0.004 & 0.022 & 0.989 & 1.000 \\
\hline 0.2 & 0.2 & 1 & 1.178 & 1.384 & 0.014 & 0.027 & 0.956 & 0.993 \\
\hline 0.2 & 0.2 & 2 & 1.455 & 2.155 & 0.034 & 0.042 & 0.794 & 0.914 \\
\hline 0.2 & 0.2 & 3 & 1.040 & 0.765 & 0.005 & 0.023 & 0.990 & 1.000 \\
\hline
\end{tabular}

Table 3. Simulation results for $q=0.75$

\begin{tabular}{|c|c|c|c|c|c|c|c|c|}
\hline \multirow[b]{2}{*}{$g$} & \multirow[b]{2}{*}{$h$} & \multirow[b]{2}{*}{ VP } & \multirow[b]{2}{*}{ RMSE } & \multirow[b]{2}{*}{ RMAX } & \multicolumn{2}{|c|}{ BIAS } & \multicolumn{2}{|c|}{$T$} \\
\hline & & & & & COBS & $\mathbf{R}$ & COBS & $\mathbf{R}$ \\
\hline 0.0 & 0.0 & 1 & 1.046 & 1.375 & -0.017 & 0.077 & 0.938 & 0.994 \\
\hline 0.0 & 0.0 & 2 & 1.328 & 2.020 & -0.052 & 0.027 & 0.709 & 0.862 \\
\hline 0.0 & 0.0 & 3 & 0.644 & 0.807 & -0.014 & 0.105 & 0.973 & 0.998 \\
\hline 0.0 & 0.2 & 1 & 0.847 & 1.459 & 0.010 & 0.137 & 0.911 & 0.978 \\
\hline 0.0 & 0.2 & 2 & 1.124 & 2.140 & 0.022 & 0.136 & 0.666 & 0.794 \\
\hline 0.0 & 0.2 & 3 & 0.544 & 0.858 & 0.001 & 0.145 & 0.969 & 0.995 \\
\hline 0.2 & 0.0 & 1 & 0.964 & 1.423 & 0.000 & 0.110 & 0.907 & 0.985 \\
\hline 0.2 & 0.0 & 2 & 1.284 & 2.057 & -0.026 & 0.074 & 0.655 & 0.803 \\
\hline 0.2 & 0.0 & 3 & 0.642 & 0.860 & -0.006 & 0.126 & 0.962 & 0.997 \\
\hline 0.2 & 0.2 & 1 & 0.849 & 1.505 & 0.042 & 0.181 & 0.880 & 0.953 \\
\hline 0.2 & 0.2 & 2 & 1.195 & 2.214 & 0.084 & 0.202 & 0.614 & 0.740 \\
\hline 0.2 & 0.2 & 3 & 0.552 & 0.945 & 0.013 & 0.167 & 0.955 & 0.993 \\
\hline
\end{tabular}




\section{RAND R. WILCOX}

\section{Illustrations}

The data in Figures 1-3 are used to illustrate method R. The left panel of Figure 4 shows the 0.5 quantile regression line for CAR and CESD. Notice that, for CAR positive (cortisol decreases after awakening), the plot suggests a positive association with depressive symptoms, which is consistent with Figure 1. But, for CAR negative, method $\mathrm{R}$ suggests that there is little or no association with CESD and clearly provides a different sense regarding the nature of the association. A criticism might be that, if method $\mathrm{R}$ were to use a smaller choice for the span, perhaps an association similar to Figure 1 would be revealed. But even with a span of $f=0.5$, the plot of the regression line is very similar to the one shown in Figure 4.

The right panel of Figure 4 shows the 0.75 quantile regression line for predicting perceived control based on CAR, which differs in an obvious way from the regression line based on COBS shown in Figure 2. Figure 4 indicates that there is little or no indication of an association with CAR when CAR is negative, but for CAR positive, a negative association is indicated. The only point is that the choice between COBS and method R can make a substantial difference.

Figure 5 shows the 0.5 quantile regression line based on the data used in Figure 3. In contrast to COBS, method $\mathrm{R}$ provides a very good approximation of the true regression line. Again, this only illustrates the extent to which the two methods can give strikingly different results. As is evident, in this particularly case, method $\mathrm{R}$ provides a much more accurate indication of the true regression line. 


\section{COMPARISONS OF TWO QUANTILE REGRESSION SMOOTHERS}
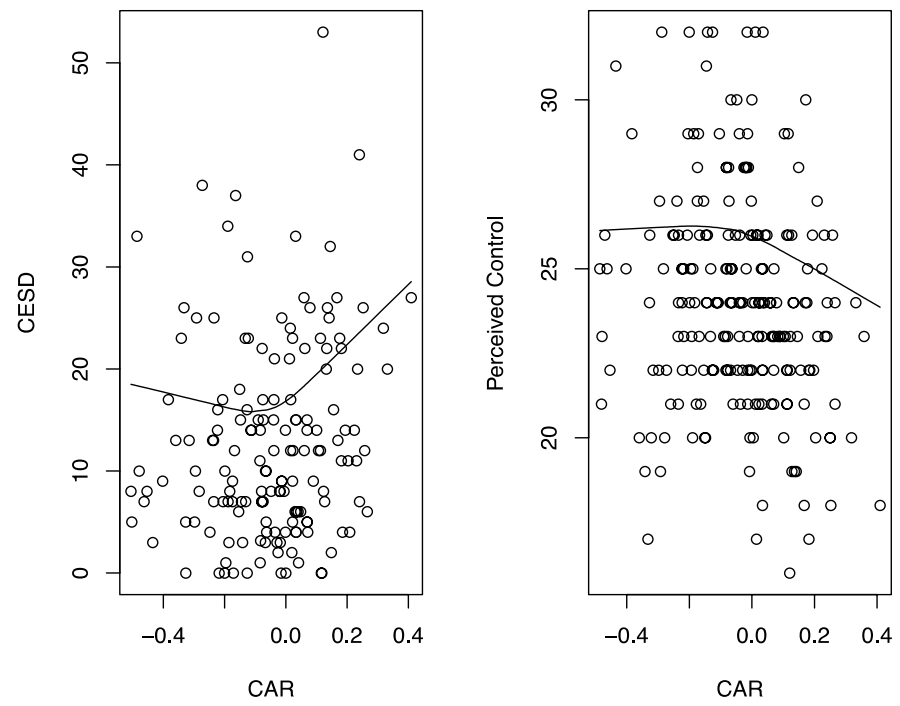

Figure 4. The quantile regression lines using method $R$ and the data in Figures 1 and 2

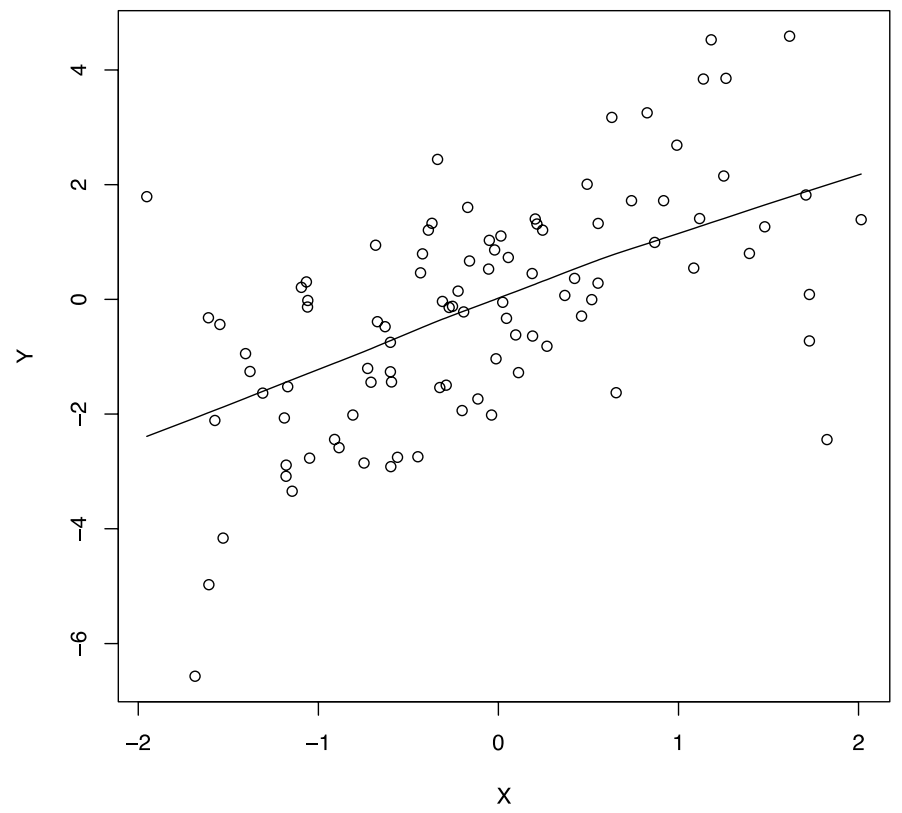

Figure 5. The quantile regression line using method $R$ and the data in Figure 3 


\section{Conclusion}

For the situations considered in the simulations, method $\mathrm{R}$ does not dominate COBS based on the four criteria used here. COBS seems to have an advantage in terms of minimizing bias. But otherwise, method R competes well with COBS, particularly in terms of Kendall's tau, which suggests that typically method $\mathrm{R}$ is better able to avoid an indication of spurious curvature. Moreover, the illustrations demonstrate that the choice between the two methods can make a substantial difference even with a sample size of $n=328$. So in summary, method $\mathrm{R}$ would seem to deserve serious consideration.

Another possible appeal of method $\mathrm{R}$ is that it is readily extended to the situation where there is more than one independent variable. That is, a generalization of the running interval smooth already exists (e.g., Wilcox, 2012). Moreover, additional smoothing can be accomplished, if desired, using the smoother derived by Cleveland and Devlin (1988), which generalizes the technique derived by Cleveland (1979). Evidently, a generalization of COBS to more than one independent variable has not been derived.

Finally, an R function for applying method R, called ghdsm, is available in the $\mathrm{R}$ package WRS, a version of which is provided as a supplemental item to this article. Updated versions may be found at dornsife.usc.edu/cf/labs/wilcox/wilcoxfaculty-display.cfm under the "Software" tab.

\section{References}

Chida, Y., \& Steptoe, A. (2009). Cortisol awakening response and psychosocial factors: A systematic review and meta-analysis. Biological Psychology, 80(3), 265-278. doi: 10.1016/j.biopsycho.2008.10.004

Clark, F., Jackson, J., Carlson, M., Chou, C., Cherry, B. J., Jordan-Marsh, M.,...Azen, S. P. (2011). Effectiveness of a lifestyle intervention in promoting the well-being of independently living older people: results of the Well Elderly 2 Randomise Controlled Trial. Journal of Epidemiology and Community Health, 66(9), 782-790. doi: 10.1136/jech.2009.099754

Cleveland, W. S. (1979). Robust locally weighted regression and smoothing scatterplots. Journal of the American Statistical Association, 74(368), 829-836. doi: 10.1080/01621459.1979.10481038 


\section{COMPARISONS OF TWO QUANTILE REGRESSION SMOOTHERS}

Cleveland, W.S., \& Devlin, S. J. (1988). Locally weighted regression: An approach to regression analysis by local fitting. Journal of the American Statistical Association, 83(403), 596-610. doi: 10.1080/01621459.1988.10478639

Clow, A., Thorn, L., Evans, P., \& Hucklebridge, F. (2004). The awakening cortisol response: Methodological issues and significance. Stress: The International Journal on the Biology of Stress, 7(1), 29-37. doi: 10.1080/10253890410001667205

Dielman, T., Lowry, C., \& Pfaffenberger, R. (1994). A comparison of quantile estimators. Communications in Statistics - Simulation and Computation, 23(2), 355-371. doi: 10.1080/03610919408813175

Efromovich, S. (1999). Nonparametric curve estimation: Methods, theory and applications. New York, NY: Springer-Verlag.

Eizenman, D. R., Nesselroade, J. R., Featherman, D. L., \& Rowe, J. W. (1997). Intraindividual variability in perceived control in an older sample: The MacArthur successful aging studies. Psychology and Aging, 12(3), 489-502. doi: 10.1037/0882-7974.12.3.489

Eubank, R. L. (1999). Nonparametric regression and spline smoothing. New York, NY: Marcel Dekker.

Foley K., Reed P., Mutran, E., \& DeVellis, R. F. (2002). Measurement adequacy of the CES-D among a sample of older African-Americans. Psychiatric Research, 109(1), 61-69. doi: 10.1016/S0165-1781(01)00360-2

Györfi, L., Kohler, M., Krzyzk, A., \& Walk, H. (2002). A distribution-free theory of nonparametric regression. New York, NY: Springer Verlag.

Härdle, W. (1990). Applied nonparametric regression. Econometric Society Monographs (No. 19). Cambridge, UK: Cambridge University Press.

Harrell, F. E., \& Davis, C. E. (1982). A new distribution-free quantile estimator. Biometrika, 69(3), 635-640. doi: 10.1093/biomet/69.3.635

He, X., \& Ng, P. (1999). COBS: Qualitative constrained smoothing via linear programming. Computational Statistics, 14(3), 315-337. doi: $10.1007 / \mathrm{s} 001800050019$

Hoaglin, D. C. (1985). Summarizing shape numerically: The $g$-and- $h$ distribution. In D. Hoaglin, F. Mosteller, \& J. Tukey (Eds.), Exploring Data Tables Trends and Shapes (pp. 461-515). New York, NY: Wiley.

Jackson, J., Mandel, D., Blanchard, J., Carlson, M., Cherry, B., Azen, S.,...Clark, F. (2009). Confronting challenges in intervention research with 


\section{RAND R. WILCOX}

ethnically diverse older adults: The USC Well Elderly II trial. Clinical Trials, 6(1), 90-101. doi: 10.1177/1740774508101191

Koenker, R., \& Bassett, G. (1978). Regression quantiles. Econometrica, 46(1), 33-50. doi: 10.2307/1913643

Koenker, R., \& Ng, P. (2005). Inequality constrained quantile regression. Sankhyā: The Indian Journal of Statistics, 67(2), 418-440. Available from http://www.jstor.org/stable/25053440

Koenker, R., Ng, P., \& Portnoy, S. (1994). Quantile smoothing splines. Biometrika, 81(4), 673-680. doi: 10.1093/biomet/81.4.673

Lewinsohn, P. M., Hoberman, H. M., \& Rosenbaum M. (1988). A prospective study of risk factors for unipolar depression. Journal of Abnormal Psychology, 97(3), 251-264. doi: 10.1037/0021-843X.97.3.251

Parrish, R. S. (1990). Comparison of quantile estimators in normal sampling. Biometrics, 46(1), 247-257. doi: 10.2307/2531649

Radloff, L. (1977). The CES-D scale: A self report depression scale for research in the general population. Applied Psychological Measurement, 1(3), 385-401. doi: 10.1177/014662167700100306

Sfakianakis, M. E., \& Verginis, D. G. (2006). A new family of nonparametric quantile estimators. Communications in Statistics - Simulation and Computation, 37(2), 337-345. doi: 10.1080/03610910701790491

Sheather, S. J., \& Marron, J. S. (1990). Kernel quantile estimators. Journal of the American Statistical Association, 85(410), 410-416. doi:

10.1080/01621459.1990.10476214

Wilcox, R. R. (2012). Introduction to robust estimation and hypothesis testing (3rd ed.). San Diego, CA: Academic Press. 